\title{
Optimal investment under transaction costs for an insurer
}

\author{
Stefan Thonhauser
}

Received: 17 December 2012/Revised: 6 September 2013/Accepted: 16 September $2013 /$

Published online: 15 October 2013

(C) DAV / DGVFM 2013

\begin{abstract}
We deal with the problem of minimizing the probability of ruin of an insurer by optimal investment of parts of the surplus in the financial market, modeled by geometric Brownian motion. In a diffusion framework the classical solution to this problem is to hold a constant amount of money in stocks, which in practice means continuous adaption of the investment position. In this paper, we introduce both proportional and fixed transaction costs, which leads to a more realistic scenario. In mathematical terms, the problem is now of impulse control type. Its solution is characterized and calculated by iteration of associated optimal stopping problems. Finally some numerical examples illustrate the resulting optimal investment policy and its deviation from the optimal investment behaviour without transaction costs.
\end{abstract}

\section{Introduction}

Over the last years the question of how to control the investment policy of an insurance company has become more important because of the strong connections between insurance and financial industries. In particular, an insurance company can no longer isolate its sources of risk and needs to deal with them from a global point of view. Therefore, setting up an appropriate-more realistic-model, the identification of crucial quantities to be controlled and solving the resulting mathematical problem is a demanding and interesting task. For example, interesting quantities in this context can be the probability of ruin, the amount of expected dividend payments, the necessary capital injections or certain risk measures such as value at risk or expected shortfall. Whereas criteria based on expected dividend payments valuate an insurance portfolio by its potential for profitability, ruin

S. Thonhauser $(\square)$

Department of Actuarial Science, HEC, University of Lausanne, 1015 Lausanne, Switzerland e-mail: stefan.thonhauser@unil.ch 
probabilities, capital injections and risk measures are a valuation of its safety. Usually, in practice risk measures are computed on a periodic basis but they can lose some clarity, when considered on larger time scales. Therefore, when looking at long term horizons, the classical probability of ruin still serves as a useful measure of solvency. In risk theory, continuous time models with stochastic return on investments or facing a stochastic environment were introduced by Paulsen [26] and Paulsen and Gjessing [27]. Following an arbitrary investment policy, Kalashnikov and Norberg [19] and Frolova et al. [11] illustrated that an exponential decay of the ruin probability (for increasing initial capital) for the portfolio without investment may change to a polynomial decay in the presence of investment. Consequently, a wrong choice of the investment policy can easily make the situation more risky. This observation gives a good motivation to formulate the problem of minimizing the probability of ruin by controlling the investment policy in the framework of stochastic optimal control theory.

Whilst in mathematical finance techniques of optimal stochastic control for solving portfolio optimization problems were introduced in the early 1970s by Merton [22, 23] and were intensely developed thereafter, it took up to 1995 until these techniques were used in an insurance context. Browne in [6] solves for the first time the problem of minimizing the probability of ruin of an underlying surplus process by means of optimal investment in a diffusion framework. Remarkable is the fact that in this model it turns out that the optimal amount of money to be invested is a constant in time, irrespective of the present level of surplus. As a starting point for the present research there will be a short illustration of this classical result in Sect. 2.

In the case of the classical compound Poisson risk model as the underlying surplus process and Black-Scholes type investment opportunity, the problem of optimal investment was solved by Hipp and Plum [14] and in a more general framework in [15]. In the presence of jumps the optimal investment policy is no longer a constant and turns out to be a function of wealth whose form depends on the type of the involved claim amount distributions. Variants of this problem are studied by Azcue and Muler [2], introducing short-selling constraints, Belkina et al. [5], under proportional short-selling and leverage constraints, or Schmidli [28], who-in addition to investment - considers the possibility of controlling through reinsurance. There are also various results on the asymptotic behaviour of the probability of ruin under an optimal investment policy, see Hipp and Schmidli [16], Gaier and Grandits [12] or Gaier et al. [13]. For an overview on the concept of ruin probabilities and various models used in risk theory the reader is referred to Asmussen and Albrecher [1]. Results on minimizing capital injections by controlling investment and reinsurance are exhibited in Eisenberg [8]. Several applications of stochastic control in risk theory and life insurance can be found in Schmidli [30].

In the present manuscript we consider the problem of maximizing the survival probability, that is one minus the probability of ruin, by controlling the investment policy if each adaptation in the investment position is afflicted by proportional and fixed transaction costs. The introduction of transaction costs is instrumental in overcoming drawbacks of the previously mentioned results, where, independent of the underlying risk model, optimal investment policies always enforce continuous trading activities. 
We are going to analyze this optimization problem in a diffusion framework. I.e., we assume a diffusion approximation of the classical compound Poisson risk model and a Black-Scholes type risky asset as the investment opportunity. This approach adds some practical aspects to the result of Browne [6] and gives some insight how optimal policies change if one can not continuously intervene anymore.

The manuscript is organized as follows: Sect. 2 introduces the considered model in mathematical terms. In Sect. 3 the maximal survival probability is characterized in terms of a limit of iterated optimal stopping problems. Furthermore the connection to the so-called quasi-variational inequalities is formalized. This section is closed with a so-called Verification Theorem. Numerical examples are given in Sect. 4. A conclusion and interpretation of the results are stated in Sect. 5.

\section{Mathematical model}

Let $\left(\Omega, \mathcal{F},\left\{\mathcal{F}_{t}\right\}_{t>0}, P\right)$ be a filtered probability space, $\left\{\mathcal{F}_{t}\right\}_{t>0}$ being complete and right-continuous. Furthermore let $W^{(1)}=\left(W^{(1)}\right)_{t \geq 0}$ and $W^{(2)}=\left(W^{(2)}\right)_{t \geq 0}$ be two independent Brownian motions on this probability space. Throughout the paper we will assume that the uncontrolled surplus process $R=\left(R_{t}\right)_{t} \geq 0$ follows the dynamics given by

$$
d R_{t}=\mu d t+\sigma d W_{t}^{(1)}, \quad \text { for } t \geq 0,
$$

where the parameters $\mu$ and $\sigma$ are positive real numbers. This type of surplus process can be interpreted as a diffusion approximation of a classical compound Poisson risk model, references for this procedure are for example Iglehart [17] or Schmidli [29]. As an extension to the classical setting, the insurer is allowed to invest some part of the surplus in a risky asset. The price process $S=\left(S_{t}\right)_{t} \geq 0$ of this asset follows the Black-Scholes model given by

$$
d S_{t}=S_{t}\left(a d t+b d W_{t}^{(2)}\right), \quad \text { for } t \geq 0,
$$

with some given initial price $S_{0}=s>0$ and parameters $a, b>0$. Furthermore we assume that the risk free rate in the present financial market setting is equal to zero. Now suppose that at time zero the insurer invests some amount $A_{0}=A \geq 0$ in the risky asset and possibly improves in this way the performance of the surplus, where the condition $A_{0} \geq 0$ represents a short-selling constraint in the considered model. The surplus including investment $T^{A}=\left(T_{t}^{A}\right)_{t} \geq 0$ has some initial value $T_{0}^{A}=x>0$ and follows:

$$
\begin{aligned}
d T_{t}^{A} & =d R_{t}+d A_{t}, \quad \text { for } t \geq 0, \\
d A_{t} & =A_{t}\left(a d t+b d W_{t}^{(2)}\right), \quad \text { for } t \geq 0 .
\end{aligned}
$$

Remark 2.1 The initial capital $x$ is the virtual sum of initial capital from the pure surplus process and the initial amount of money invested, i.e. $x=R_{0}+A_{0}$. Since it is assumed that the risk-free rate is equal to zero, one does not need to split up the dynamics of $T^{A}$ into a proportion invested in the risky asset and a proportion 
invested in a risk-free asset. This can be seen as follows, suppose a fraction $0 \leq p \leq 1$ of the present wealth is invested in the risky asset and the fraction $1-p$ is invested in a bond with interest rate $r$. Then the wealth $\left(T_{t}\right)_{t} \geq 0$ follows,

$$
d T_{t}=d R_{t}+p T_{t}\left(a d t+b d W_{t}^{(2)}\right)+(1-p) r T_{t} d t, \quad T_{0}=x .
$$

Since $r=0$ we have that $p T_{t}$ equals the amount of money invested at time $t$, which is equal to $A_{t}$ and evolves like $d A_{t}=A_{t}\left(a d t+b d W_{t}^{(2)}\right)$.

The initial investment is $A=N S_{0}$, where $N \geq 0$ is the number of shares initially bought, consequently at time $t$ without any intervention $A_{t}=N S_{t}$.

The time of ruin of $T^{A}$ is given by $\tau^{A}=\inf \left\{t \geq 0 \mid T_{t}^{A}<0\right\}$, because of the presence of Brownian motions we have $\tau^{A}=\inf \left\{t \geq 0 \mid T_{t}^{A} \leq 0\right\}$ with probability one.

The associated probability of survival is $\varphi^{0}(x, A)=P_{x, A}\left(\tau^{A}=\infty\right)$. A classical result states that in this specific setting we have $\varphi^{0}(x, 0)=1-e^{-\frac{2 \mu}{\sigma^{2}} x}$, cf. [1]. In addition we have the following (asymptotic) boundary conditions:

$$
\begin{array}{ll}
\lim _{x \rightarrow \infty} \varphi^{0}(x, A)=1, & \text { for fixed } A \geq 0, \\
\lim _{A \rightarrow \infty} \varphi^{0}(x, A)=0, & \text { for fixed } x>0,
\end{array}
$$

the second one is due to the unbounded weight of the investment's Brownian motion part of the evolution of $T^{A}$.

Up to now we defined the model to have a starting investment position (which may be there due to some policies in the past). From now on we will allow the insurer to modify the amount of money exposed to investment, but each change will be subject to transaction costs:

$$
\text { a change } A \rightarrow A+\Delta A \quad \text { leads to costs } K+k|\Delta A|,
$$

where $K>0$ is a fixed cost and $k>0$ represents a proportional cost factor. Since a continuous change of the investment position would lead to unbounded transaction costs it is natural to consider impulse controls as the class of admissible controls in the resulting optimization problem. The set of admissible investment policies is fixed in the following definition, see also Korn [20].

Definition 2.1 An admissible investment policy is of the form $\pi=\left\{\left(\theta_{n}, A_{n}\right)\right\}_{n \in \mathbb{N}^{N}} \cdot \theta_{i}$ is an intervention time and $A_{i}$ denotes the investment's new position,

1. $0 \leq \theta_{n} \leq \theta_{n+1}$ a.s. for all $n \in \mathbb{N}$

2. $\theta_{n}$ is a stopping time w.r.t. the filtration $\sigma\left\{\left(W_{s}^{(1)}, W_{s}^{(2)}\right), s \leq t,\left(\theta_{k}, A_{k}\right), k<n\right\}$ and $\theta_{n} \leq \tau^{\pi}$ for all $n \in \mathbb{N}$

3. $A_{n} \geq 0$ is measurable with respect to $\sigma\left\{\left(W_{s}^{(1)}, W_{s}^{(2)}\right), s \leq t,\left(\theta_{k}, A_{k}\right), k<n\right\}$

4. $P\left(\left\{\lim _{n \rightarrow \infty} \theta_{n} \leq T\right\} I_{\left\{T<\tau^{\pi}\right\}}\right)=0$ for all $T>0$ and $\lim _{n \rightarrow \infty} \theta_{n}=\tau^{\pi}$ a.s.

The set of admissible investment policies is denoted by $\Pi$.

The requirement $\theta_{n} \leq \tau^{\pi}$ in (2) can also be found in $\varnothing \mathrm{ksendal}$ and Sulem [25], here $\tau^{\pi}$ denotes the time of ruin for the controlled process. It says that if ruin occurs, 
the sequence becomes constant from that time onwards with no effect on the (then stopped) process. In point (3), $A_{n} \geq 0$ specifically prohibits short-selling of the risky asset. Point (4) gives that on bounded time intervals, before the event of ruin, there are only finitely many interventions.

Let $\pi=\left\{\left(\theta_{n}, A_{n}\right)\right\}_{n \in \mathbb{N}}$ be an admissible policy, the controlled surplus process $T^{\pi}=\left(T_{t}^{\pi}\right)_{t} \geq 0$ is then given by

$$
\begin{aligned}
T_{t}^{\pi}= & x+\int_{0}^{t} d R_{s}+\sum_{n=1}^{\infty} \int_{\theta_{n-1} \wedge t}^{\theta_{n} \wedge t} A_{s}^{\pi}\left(a d s+b d W_{s}^{(2)}\right) \\
& -\sum_{n=1}^{\infty}\left(K+k\left|\Delta A_{\theta_{n}}^{\pi}\right|\right) I_{\left\{\theta_{n} \leq t\right\}} \\
d A_{s}^{\pi}= & A_{s}^{\pi}\left(a d s+b d W_{s}^{(2)}\right), \quad \text { for } \theta_{n-1} \leq s<\theta_{n} \\
A_{\theta_{n}}^{\pi}= & A_{\theta_{n}-}^{\pi}+\Delta A_{\theta_{n}}^{\pi}=A_{n}, \\
A_{0}^{\pi}= & A .
\end{aligned}
$$

Now formally we can define $\tau^{\pi}=\inf \left\{t \geq 0 \mid T_{t}^{\pi} \leq 0\right\}$ and

$$
\varphi^{\pi}(x, A)=P_{x, A}\left(\tau^{\pi}=\infty\right)=\mathbb{E}_{x, A}\left(I_{\left\{\tau^{\pi}=\infty\right\}}\right),
$$

the associated survival probability. The value function is the maximal survival probability which can be achieved by using an admissible investment policy,

$$
\varphi(x, A)=\sup _{\pi \in \Pi} \varphi^{\pi}(x, A) .
$$

From the statement of the optimization problem and the structure of the transaction costs, it is apparent that it is necessary to know the investment position when deciding on an intervention.

Before going on with a characterization of the value function $\varphi(x, A)$ of the optimization problem in Sect. 3, we review some results of Browne [6] and present some analysis of $\varphi^{0}(x, A)$.

\subsection{The problem without transaction costs}

The following results are due to Browne [6] and can also be found in Schmidli [30]. In the setup without transaction costs, there is no need to remember the investment's position (i.e. the number of shares), and an admissible policy is given by a càdlàg process $A=\left(A_{t}\right)_{t} \geq 0$ which is adapted to the filtration generated by the Brownian motions and allows the existence of a unique solution to

$$
d X_{t}^{A}=\left(\mu+a A_{t}\right) d t+\sigma d W_{t}^{(1)}+b A_{t} d W_{t}^{(2)}, \quad X_{0}^{A}=x>0 .
$$

This stochastic differential equation describes the evolution of the surplus using policy $A$. Let $\tau^{A}=\inf \left\{t \geq 0 \mid X_{t}^{A} \leq 0\right\}$ denote the time of ruin within this model and set 


$$
\varphi^{B}(x)=\sup _{A} P_{x}\left(\tau^{A}=\infty\right),
$$

to be the largest possible survival probability. Using the dynamic programming approach one gets that

$$
\varphi^{B}(x)=1-e^{-\eta x}, \quad x \geq 0,
$$

where

$$
\eta=\frac{1}{\sigma^{2} b}\left(\mu b+\sqrt{\mu^{2} b^{2}+a^{2} \sigma^{2}}\right) .
$$

The optimal investment policy $A^{*}$ turns out to be a constant

$$
A^{*}=\frac{a}{b^{2} \eta}>0 \text {. }
$$

The fact that $A^{*}$ is constant has the effect that it is always optimal to hold exactly an investment position of size $A^{*}$, and because of the presence of Brownian motion one needs to continuously buy and sell fractions of the risky asset to maintain this position. From (3) we make the following observations:

- $A^{*}$ is increasing in $\sigma>0$,

- $A^{*}$ is decreasing in $b>0$, with $\lim _{b \rightarrow 0} A^{*}=\infty$.

We can interpret these facts as follows: one needs to invest more if the risk from the insurance business is serious. On the other hand, if the asset's risk is too large one is well advised to reduce the position, which is quite natural for reducing the probability of ruin. We will also observe this type of behaviour later for the problem with transaction costs in Sect. 4.

\subsection{Study of $\varphi^{0}$}

In this section we are going to study the (bivariate) survival probability $\varphi^{0}(x, A)$ if there are no changes in the investment position. The results will also serve as a theoretical basis for an iteration procedure which is part of the control problem stated in Sect. 3. As mentioned above, $\varphi^{0}(x, A)$ fulfills the following boundary conditions:

$$
\left\{\begin{array}{cl}
\varphi^{0}(0, A)=0 \quad A \geq 0, & \\
\varphi^{0}(x, 0)=1-e^{-\frac{2 \mu}{\sigma^{2}} x} & x \geq 0, \\
\lim _{x \rightarrow \infty} \varphi^{0}(x, A)=1 & \text { for fixed } A \geq 0 \\
\lim _{A \rightarrow \infty} \varphi^{0}(x, A)=0 & \text { for fixed } x>0 .
\end{array}\right.
$$

Now assume that $\varphi^{0} \in \mathcal{C}^{2}([0 \infty) \times[0, \infty))$ and set $\varphi^{0}(x, A)=0$ if $x \leq 0$. A conditioning argument on a small time interval and Itô's formula suggest that $\varphi^{0}(x, A)$ is a solution to the following partial differential equation:

$$
\begin{aligned}
0= & \mathcal{L} w(x, A):=(\mu+A a) w_{x}(x, A)+\left(\frac{\sigma^{2}}{2}+\frac{A^{2} b^{2}}{2}\right) w_{x x}(x, A) \\
& +A^{2} b^{2} w_{x A}(x, A)+\operatorname{Aaw}_{A}(x, A)+\frac{A^{2} b^{2}}{2} w_{A A}(x, A) .
\end{aligned}
$$


Unfortunately it is very hard, if not impossible, to find an explicit expression for $\varphi^{0}$. Therefore we have to use a numerical method for studying this survival probability. At first we need to bound the domain of $\varphi^{0}$ and look at a related problem.

Let $\bar{x}>0$ and $\bar{A}>0$, define $\tau_{\bar{x}}=\inf \left\{t \geq 0 \mid T_{t}^{A}=\bar{x}\right\}$ and $\tau_{\bar{A}}=\inf \left\{t \geq 0 \mid A_{t}=\right.$ $\bar{A}\}$. Introduce $\bar{\varphi}(x, A)=P_{x, A}\left(\tau_{\bar{x}}<\tau_{\bar{A}} \wedge \tau^{A}\right)$, the probability when starting in $(x, A)$ of hitting $\bar{x}$ before ruin or hitting $\bar{A}$.

From its definition we immediately get:

$$
\begin{cases}\bar{\varphi}(0, A)=0 & A \in[0, \bar{A}], \\ \bar{\varphi}(x, 0)=\frac{1-e^{-\frac{2 \mu}{\sigma^{2}}}}{1-e^{-\frac{\mu_{\bar{T}}}{\sigma^{2}}}} & x \in[0, \bar{x}], \\ \bar{\varphi}(\bar{x}, A)=1 & A \in[0, \bar{A}], \\ \bar{\varphi}(x, \bar{A})=0 & x \in[0, \bar{x}),\end{cases}
$$

and that $\bar{\varphi}(x, A) \rightarrow \varphi^{0}$ as $\bar{A}$ and $\bar{x}$ tend to infinity.

One may notice that from the definition we actually have that $\bar{\varphi}(\bar{x}, \bar{A})=1$, which gives that the boundary values are not continuous in the upper-right corner.

The basic idea is to first study $\bar{\varphi}$ and then choose $\bar{x}$ and $\bar{A}$ large enough such that it serves as an approximation of $\varphi^{0}$, cf. Proposition 2.3 and Corollary 2.4 from [1] for the one-dimensional case.

The following Lemma states the connection to the equation $\mathcal{L} w(x, A)=0$.

Lemma 2.2 For given fixed $\bar{x}, \bar{A}$, the function $\bar{\varphi}$ is the unique classical solution of the partial differential equation $\mathcal{L} w(x, A)=0$ with the boundary conditions (6).

Proof At first we are going to prove that this differential equation has an unique solution $g$, in a second step we show that $g=\bar{\varphi}$.

We face an elliptic operator $\mathcal{L}$, since the coefficients matrix is positive definite. From Evans [9, p. 301 and p. 316] and observing that the involved coefficients are polynomials, we get that there exists a unique solution to the problem which is at least twice differentiable in all variables on $(0, \bar{x}) \times(0, \bar{A})$. Furthermore, cf. Jost $[18$, p. 59] we can also obtain that the discretized version of the problem converges uniformly to the correct solution if the grid size tends to zero.

Now for $(x, A) \in(0, \bar{x}) \times(0, \bar{A})$ we apply Itô's formula to $g\left(T_{t \wedge \zeta}^{A}, A_{t \wedge \zeta \zeta}\right)$, with $\zeta=\min \left\{\tau_{\bar{x}}, \tau_{\bar{A}}, \tau^{A}\right\}$ the first exit time from $(0, \bar{x}) \times(0, \bar{A})$,

$$
\begin{aligned}
g\left(T_{t \wedge \zeta}^{A}, A_{t \wedge \zeta}\right)= & g(x, A)+\int_{0}^{t \wedge \zeta} \mathcal{L} g\left(T_{s}^{A}, A_{s}\right) d s \\
& +\int_{0}^{t \wedge \zeta} g_{x}\left(T_{s}^{A}, A_{s}\right) \sigma d W_{s}^{(1)}+\int_{0}^{t \wedge \zeta} g_{A}\left(T_{s}^{A}, A_{s}\right) b A_{s} d W_{s}^{(2)} .
\end{aligned}
$$

Since $\mathcal{L} g=0$ on $(0, \bar{x}) \times(0, \bar{A})$ and the involved stochastic integrals are zero-mean martingales, we can state that 


$$
\mathbb{E}_{x, A}\left(g\left(T_{t \wedge \zeta}^{A}, A_{t \wedge \zeta}\right)\right)=g(x, A) .
$$

Now we use $P_{x, A}(\zeta<\infty)=1$ and exploit the stated boundary conditions, from bounded convergence we can deduce the statement of the Lemma when $t \rightarrow \infty$ in Eq. (7),

$$
g(x, A)=\mathbb{E}_{x, A}\left(g\left(T_{\zeta}^{A}, A_{\zeta}\right)\right)=\mathbb{E}_{x, A}\left(I_{\left\{\tau_{\bar{x}}<\tau_{\bar{A}} \wedge \tau^{A}\right\}}\right)=\bar{\varphi}(x, A) .
$$

By the end of the section we collect properties of the survival probability of the uncontrolled process $\left(T^{A}, A\right)$. From its definition we have that it fulfills the boundary conditions given in (4). Assume two initial capital values $x>x^{\prime}$ as starting points of paths of $T^{A}$ with the same investment component. Naturally, because of the continuity of the paths, ruin occurs first for the one starting in $x^{\prime}$, therefore $\varphi^{0}(x, A) \geq \varphi^{0}\left(x^{\prime}, A\right)$, i.e. $\varphi^{0}$ is increasing in the $x$ component.

Because of the Markov property of the bivariate process $\left(T^{A}, A\right)$ the survival probability $\varphi$ is a so-called $\left(T^{A}, A\right)$-harmonic function, see [24, Definition 9.2.2.], as a consequence we can state the following Lemma.

Lemma 2.3 On every compact subset $M$ of $(0, \infty) \times(0, \infty)$ we have that for all $\alpha<1, \varphi^{0}(x, A)=P_{x, A}\left(\tau^{A}=\infty\right)$ admits $\alpha$-Hölder continuous second order derivatives and $\mathcal{L} \varphi^{0}=0$.

Proof We observe that due to the Markov property of $\left(T_{t}^{A}, A_{t}\right)_{t} \geq 0$ we have

$$
\varphi^{0}(x, A)=\mathbb{E}_{x, A}\left(\varphi^{0}\left(T_{\tau_{U}}^{A}, A_{\tau_{U}}\right)\right),
$$

with $\tau_{U}=\inf \left\{t \geq 0 \mid\left(T_{t}^{A}, A_{t}\right) \in \delta U\right\}$, for all bounded open sets $U \subset(0, \infty) \times$ $(0, \infty)$ such that $(x, A) \in U$. Now we can conclude from the Hölder continuity of the first exit distribution of $\left(T_{t}^{A}, A_{t}\right)$ from an open ball, shown in the proof of Lemma 9.2.14. in Øksendal [24], that $\varphi^{0}(x, A)$ is Hölder continuous on compacts as well (the above expectation can be written as an integral with respect to this special measure).

The rest of the proof follows arguments used in the first part of the proof of [Lemma 9.2.14., 24], which we will sketch in the following. Again on an open ball $B(x, A)$ we can look at the Dirichlet problem given by

$$
\mathcal{L} w=0 \quad \text { on } B(x, A) \quad \text { and } \quad w=\varphi^{0} \quad \text { on } \delta B(x, A),
$$

which allows for an unique classical solution $u$ with Hölder continuous second order derivatives on $B(x, A)$. Applying Itô's formula to $u\left(T_{t}^{A}, A_{t}\right)$, as done in the proof of Lemma 2.2, yields $\varphi^{0}=u$ on $B(x, A)$ and the statement of the Lemma follows.

Before going on with the optimization problem we give some numerical examples for $\bar{\varphi}$. Figure 1 illustrates $\bar{\varphi}(x, A)$ on $[0,5] \times[0,10]$ for the following choice of parameters (Parameter set 1): 


\begin{tabular}{lllll}
\hline$\mu$ & $\sigma$ & $a$ & $b$ & $A^{*}$ \\
\hline 0.3 & 0.4 & 0.2 & 0.4 and 0.6 & 0.304 and 0.1415 \\
\hline
\end{tabular}

The left-hand graphic shows $\bar{\varphi}$ for the lower value of the risky asset's volatility, whereas the right-hand graphic shows $\bar{\varphi}$ for the larger volatility. The two graphics in Fig. 2 cover $\bar{\varphi}$ for an increased volatility in the insurance business and again for high and low asset's volatility. Concretly we use the parameters (Parameter set 2):

\begin{tabular}{lllll}
\hline$\mu$ & $\sigma$ & $a$ & $b$ & $A^{*}$ \\
\hline 0.3 & 0.8 & 0.2 & 0.4 and 0.6 & 1 and 0.507 \\
\hline
\end{tabular}

The choice of $\bar{x}=5$ is justified by the observation that $1-e^{-\frac{2 \mu}{\sigma^{2}} \bar{x}} \approx 1$. The choice of $\bar{A}=20$ is reasonable, since it allows, compared with the magnitude of the initial capital $x$, for a lot of different investment positions and is large enough to accept $\bar{\varphi}$ as an approximation of $\varphi^{0}$ for $0 \leq A \leq 12$. Obviously for larger values of $A$ the boundary influence $\varphi^{0}(x, \bar{A})=0$ is too dominant.

Comparing left-hand and right-hand side in Fig. 1, one can see that the increase of $b$ has a big influence on the survival probability approximated by $\bar{\varphi}$. In particular when starting, under high volatility without any further intervention, in a position $(x, A)$ where the investment component is far away from $A^{*}$ one faces a large disadvantage compared with initial investment positions close to the optimal one for the problem without transaction costs.

Looking at Figs. 1 and 2, one gets that the second set of parameters creates a riskier environment than the first set. In Fig. 2 the drawback of a wrong initial investment position is even more apparent. Obviously increasing $\sigma$, the insurer's volatility, has a severe negative effect on the survival probability such that determining an approriate investment position becomes much more important. All of the four graphics illustrate the results from Kalashnikov and Norberg [19] in a very clear way, i.e. away from the optimal investment position $A^{*}$ the rate of increase as a function in $x$ reduces considerably.

\section{Solution approach}

In this section we shall prepare the theoretical basis for the construction of the value function (2). One way to characterize $\varphi(x, A)$ is to view it as a limit of a sequence of solutions to optimal stopping problems, as done in Sect. 3.1. Another way of characterization is to derive first in a heuristic way the associated quasi-variational inequalities and then to prove that $\varphi$ is (in some sense) a solution to them. The 

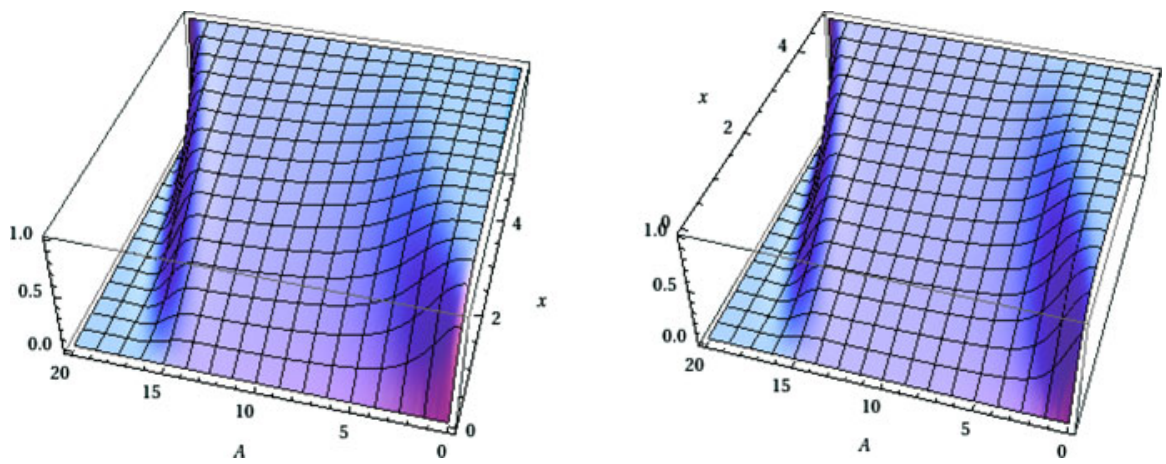

Fig. $1 \varphi^{0}$ for Parameter set 1
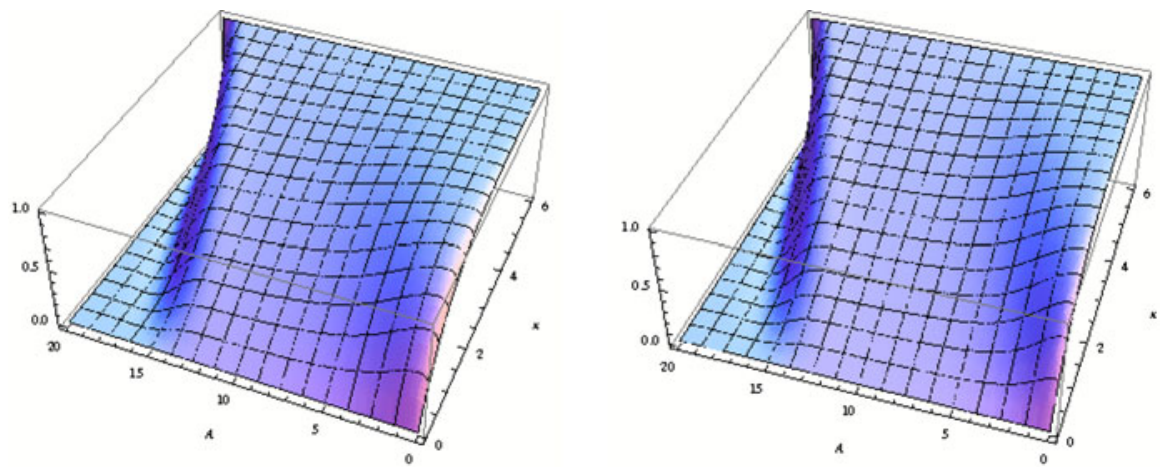

Fig. $2 \varphi^{0}$ for Parameter set 2

combination of these two approaches will be helpful to derive an approximation for the value function. In the same way as for the value function itself, one can get quasi-variational inequalities for the involved optimal stopping problems. An iteration procedure then generates an approximation of $\varphi$.

\subsection{Iterated optimal stopping}

As mentioned above, we will start with characterizing $\varphi$ through a sequence of optimal stopping problems.

Suppose a function $f:[0, \infty) \times[0, \infty) \rightarrow[0,1]$ measures the outcome of an intervention. If it would be optimal to intervene at a point $(x, A)$ we would choose the new investment position such that the continuation value, measured by $f$, is maximized, i.e.

$$
\sup _{\{\Delta A \in \mathcal{D}(x, A)\}}\{f(x-K-k|\Delta A|, A+\Delta A)\}=: M f(x, A),
$$


where $\mathcal{D}(x, A)=\{\Delta A|x-K-k| \Delta A \mid \geq 0 \wedge A+\Delta A \geq 0\}$ is the set of admissible interventions at the position $(x, A)$. If $\mathcal{D}(x, A)=\emptyset$ we define $M f(x, A)=0$, in particular for $x<0$ and $0 \leq x<K$ there is no admissible intervention.

Lemma 3.1 Suppose $f:[0, \infty) \times[0, \infty) \rightarrow[0,1]$ is increasing in $x$, continuous, $\lim _{x \rightarrow \infty} f(x, A)=1$ and $\lim _{A \rightarrow \infty} f(x, A)=0$, then all these properties translate to Mf as a function in $(x, A)$.

Proof From $0 \leq f \leq 1$ we have immediately $0 \leq M f \leq 1$. For fixed $A$ we can choose for large enough $x$, some $\Delta A \in \mathcal{D}(x, A)$, and we have

$$
f(x-K-k|\Delta A|, A+\Delta A) \leq M f(x, A) \leq 1 .
$$

Since the lower bound tends to one for $x \rightarrow \infty$ we derive the statement for $M f$.

On the other hand fix $x$ such that $\mathcal{D}(x, A) \neq \emptyset$ and choose $\Delta A^{\varepsilon}$ such that

$$
0 \leq M f(x, A) \leq f\left(x-K-k\left|\Delta A^{\varepsilon}\right|, A+\Delta A^{\varepsilon}\right)+\varepsilon,
$$

for some $\varepsilon>0$. Since for an admissible intervention we have $\frac{x-K}{k} \geq|\Delta A|$, a large investment position $A$ can not be switched off. Therefore because of continuity and the limiting behaviour of $f$ we can take $A$ large such that the right-hand side in (8) becomes arbitrarily small. This yields $\lim _{A \rightarrow \infty} M f(x, A)=0$.

Now let $x>y>0$,

$$
\begin{aligned}
& M f(x, A)-M f(y, A) \\
& \quad=\sup _{\{\Delta A \in \mathcal{D}(x, A)\}} f(x-K-k|\Delta A|, A+\Delta A)-\sup _{\left\{\Delta A^{\prime} \in \mathcal{D}(y, A)\right\}} f\left(y-K-k\left|\Delta A^{\prime}\right|, A+\Delta A^{\prime}\right),
\end{aligned}
$$

from $x>y$ we have $\mathcal{D}(x, A) \supset \mathcal{D}(y, A)$. Therefore, by choosing $\Delta A^{\varepsilon}$, some $\varepsilon$-optimal intervention for $(y, A)$, we arrive at

$$
\begin{aligned}
& \operatorname{Mf}(x, A)-M f(y, A) \\
& \quad \geq f\left(x-K-k\left|\Delta A^{\varepsilon}\right|, A+\Delta A^{\varepsilon}\right)-f\left(y-K-k\left|\Delta A^{\varepsilon}\right|, A+\Delta A^{\varepsilon}\right)-\varepsilon .
\end{aligned}
$$

Since $f$ is assumed to be monotone increasing in $x$, the result follows.

For proving continuity we first assume that for $(x, A),\left(x^{\prime}, A^{\prime}\right)$ we have $\mathcal{D}(x, A)=$ $\mathcal{D}\left(x^{\prime}, A^{\prime}\right)=\emptyset$, then $\left.M f(x, A)=M f\left(x^{\prime}, A^{\prime}\right)\right)=0$.

Now assume that both sets are non-empty. Take an $\varepsilon / 2$-optimal intervention $\Delta A^{\varepsilon}$ for $(x, A)$ and an admissible $\Delta \widetilde{A}$, to be specified below, for $\left(x^{\prime}, A^{\prime}\right)$. We get

$$
\begin{aligned}
& M f(x, A)-M f\left(x^{\prime}, A^{\prime}\right) \\
& \quad \leq f\left(x-K-k\left|\Delta A^{\varepsilon}\right|, A+\Delta A^{\varepsilon}\right)-f\left(x^{\prime}-K-k|\Delta \widetilde{A}|, A^{\prime}+\Delta \widetilde{A}\right)+\frac{\varepsilon}{2} .
\end{aligned}
$$

Looking at the structure of reachable points by an intervention $(x-K-k|\Delta A|, A+$ $\Delta A)$ for $\Delta A \in \mathcal{D}(x, A)$, one observes that these are linear translations of the starting point $(x, A)$. Therefore, for a fixed choice of $\Delta A^{\varepsilon}$, one can choose a $\Delta \widetilde{A}$ such that if $d\left((x, A),\left(x^{\prime}, A^{\prime}\right)\right)<\delta$ for a suitable $\delta>0$ we have $d\left(\left(x-K-k\left|\Delta A^{\varepsilon}\right|, A+\right.\right.$ $\left.\left.\Delta A^{\varepsilon}\right),\left(x^{\prime}-K-k|\Delta \widetilde{A}|, A^{\prime}+\Delta \widetilde{A}\right)\right)$ is small enough to get by continuity of $f$ 


$$
\left|f\left(x-K-k\left|\Delta A^{\varepsilon}\right|, A+\Delta A^{\varepsilon}\right)-f\left(x^{\prime}-K-k|\Delta \widetilde{A}|, A^{\prime}+\Delta \widetilde{A}\right)\right|<\frac{\varepsilon}{2} .
$$

Changing the role of the two points shows continuity.

Now suppose $\mathcal{D}(x, A) \neq \emptyset$ and $\mathcal{D}\left(x^{\prime}, A^{\prime}\right)=\emptyset$, as the points should be close they need to be of the form $\left(K+\delta, A\right.$, ) and $\left(K, A^{\prime}\right)$, for some $\delta>0$. This implies that an intervention at the first point shifts the $x$ argument to a value smaller than $\delta$. Since $f(0, A)=0$ for any $A \geq 0$ we get by continuity of $f$ that $0 \leq M f(x, A)$ becomes arbitrarily small for $\delta \rightarrow 0, \lim _{\delta \rightarrow 0} M f(K+\delta, A)=0$.

The next step towards a characterization of $\varphi$ is the definition of an appropriate operator, which, repeatedly applied to $\varphi^{0}$, generates a sequence of functions which tends to $\varphi$.

Suppose it is never optimal to adapt the investment position part of $T^{A}$, which happens if $\theta_{1}=\tau$, then the corresponding survival probability is equal to $\varphi^{0}$. If it is optimal to intervene before the event of ruin, one would choose the intervention time such that $\mathbb{E}_{x, A}\left(M f\left(T_{\theta}^{A}, A_{\theta}\right)\right)$ is maximized. Therefore define

$$
\mathcal{G} f(x, A):=\max \left\{\varphi^{0}(x, A), \sup _{\theta<\tau} \mathbb{E}_{x, A}\left(M f\left(T_{\theta}^{A}, A_{\theta}\right)\right)\right\},
$$

where $f$ is a function with properties as used in Lemma 3.1. For staying in the same class of functions it is important that properties of $f$ translate to properties of $\mathcal{G} f$.

Lemma 3.2 Suppose $f:[0, \infty) \times[0, \infty) \rightarrow[0,1]$ is increasing in $x$, continuous and $\lim _{x \rightarrow \infty} f(x, A)=1$, then all these properties translate to $\mathcal{G} f$ as a function in $(x, A)$.

Proof Because both terms in the maximum in (9) are between zero and one we have $0 \leq \mathcal{G} f \leq 1$.

Take $x \geq x^{\prime}$, at first observe $\varphi^{0}(x, A) \geq \varphi^{0}\left(x^{\prime}, A\right)$ for every $A \geq 0$. For the second part we have that every path of $T^{A}$ starting in $(x, A)$ is bigger or equal to the path starting in $\left(x^{\prime}, A\right)$, since the investment component is the same. Therefore the choice of an $\varepsilon$-optimal intervention and stopping time for $\sup _{\theta<\tau} \mathbb{E}_{x^{\prime}, A}\left(M f\left(T_{\theta}^{A}, A_{\theta}\right)\right.$, which is also admissible for $\sup _{\theta<\tau} \mathbb{E}_{x, A}\left(M f\left(T_{\theta}^{A}, A_{\theta}\right)\right.$, leads to $\mathcal{G} f(x, A) \geq \mathcal{G} f\left(x^{\prime}, A\right)$ because of the monotonicity in the $x$ component of $M f$.

The $\operatorname{limit}_{\lim _{x \rightarrow \infty}} \mathcal{G} f(x, A)=1$ follows immediately from $\lim _{x \rightarrow \infty} \varphi^{0}(x, A)=1$ and $\sup _{\theta<\tau} \mathbb{E}_{x, A}\left(M f\left(T_{\theta}^{A}, A_{\theta}\right) \leq 1\right.$.

The continuity of $\mathcal{G} f$ can be deduced by Theorem 8 and Lemma 5 of Krylov [21, p. 133 and p. 156]. It can also be seen by the following explicit construction, cf. Øksendal [24, Lemma 8.1.4] or Korn [20]. Let

$$
h_{0}(x, A)=M f(x, A) .
$$

Define $\mathcal{S}_{n}:=\left\{k 2^{-n}, 0 \leq k \leq 4^{n}\right\}$ for $n \in \mathbb{N}$ and

$$
h_{n}(x, A)=\max _{t \in \mathcal{S}_{n}} \mathbb{E}_{x, A}\left(h_{n-1}\left(T_{A}, A_{t}\right)\right) .
$$

Since $h_{0}$ is continuous, also $h_{1}$ is continuous, as it is a maximum out of finitely many continuous functions. By induction it follows that $h_{n}$ is continuous as well. From 
[24] we have $v \geq h_{n} \geq h_{n-1}$ and $\lim _{n \rightarrow \infty} h_{n}(x, A)=v(x, A):=\sup _{\theta \leq \tau} \mathbb{E}_{x, A}$ $\left(M f\left(T_{\theta}^{A}, A_{\theta}\right)\right)$ with $v(x, A)=0$ for $x \leq 0$. Since on $\{\theta=\tau\}$ we face the basic survival probability, it will not play any role to use $\theta<\tau$ below.

Now suppose that $\mathcal{G} f(x, A) \geq \mathcal{G} f\left(x^{\prime}, A^{\prime}\right)$, then

$$
\begin{aligned}
& 0 \leq \mathcal{G} f(x, A)-\mathcal{G} f\left(x^{\prime}, A^{\prime}\right) \leq \max \left\{\varphi^{0}(x, A), v(x, A)\right\}-\max \left\{\varphi^{0}\left(x^{\prime}, A^{\prime}\right), v\left(x^{\prime}, A^{\prime}\right)\right\} \\
& \varepsilon+\max \left\{\varphi^{0}(x, A), h_{n}(x, A)\right\}-\max \left\{\varphi^{0}\left(x^{\prime}, A^{\prime}\right), h_{n}\left(x^{\prime}, A^{\prime}\right)\right\}
\end{aligned}
$$

with $n$ such that $v(x, A)-h_{n}(x, A) \leq \varepsilon$ for some $\varepsilon>0$. Since both functions in the maximum are continuous, the difference tends to $\varepsilon$ as $(x, A) \rightarrow\left(x^{\prime}, A^{\prime}\right)$. Interchanging the role of the two points when using the approximation then gives continuity.

Now we are ready to define the iteration procedure. Define a sequence of functions $\left\{\varphi_{n}\right\}_{n \in \mathbb{N}}$ with $\varphi_{n}:[0, \infty) \times[0, \infty) \rightarrow[0,1]$ by the following recursion,

$$
\varphi_{n}(x, A):=\max \left\{\varphi^{0}(x, A), \sup _{\theta<\tau} \mathbb{E}_{x, A}\left(M \varphi_{n-1}\left(T_{\theta}^{A}, A_{\theta}\right)\right)\right\}=\mathcal{G} \varphi_{n-1}(x, A)
$$

where the starting point is given by $\varphi_{0}=\varphi^{0}$. Let $\Pi_{n} \subset \Pi$ be the set of admissible policies with at most $n \in \mathbb{N}$ interventions. Notice that from Lemma 3.2 and an induction argument we have that $\varphi_{n}$ has the same properties as $\varphi^{0}$.

We can state the first characterization of $\varphi$ as a limit of iterated optimal stopping problems.

Theorem 3.3 For the sequence defined above we have:

$$
\varphi_{n}(x, A)=\sup _{\pi \in \Pi_{n}} \varphi^{\pi}(x, A) \text { and } \quad \lim _{n \rightarrow \infty} \varphi_{n}=\varphi .
$$

Proof From its definition we have that

$$
\varphi_{1}(x, A)=\mathcal{G} \varphi^{0}(x, A) \geq \varphi^{0} .
$$

Since in $\mathcal{G} \varphi^{0}$ at most one intervention is done and in the case of an intervention it is carried out in a maximizing way, we get

$$
\varphi_{1}(x, A)=\sup _{\pi \in \Pi_{1}} \varphi^{\pi}(x, A) .
$$

Now assume

$$
\varphi_{n-1}(x, A)=\sup _{\pi \in \Pi_{n-1}} \varphi^{\pi}(x, A)=: v_{n-1}(x, A) .
$$

Since $\Pi_{n-1} \subset \Pi_{n}$ we have $\varphi_{n-1}=v_{n-1} \leq v_{n} \leq \varphi$ and because when calculating $\varphi_{n}$ at most $n$ interventions appear $\varphi_{n} \leq v_{n}$ holds.

Now let $\left.\pi=\left\{\left(\theta_{1}, A_{1}\right), \ldots,\left(\theta_{n}, A_{n}\right)\right)\right\} \in \Pi_{n}$, for the associated survival probability we have

$$
\varphi^{\pi}(x, A)=\mathbb{E}_{x, A}\left(P_{T^{*}, A_{1}}\left(T_{t}^{\pi}>0 \text { for all } t \geq \theta_{1}\right) I_{\left\{\theta_{1}<\tau\right\}}+\varphi^{0}(x, A) I_{\left\{\theta_{1}=\tau\right\}}\right),
$$


where $T^{*}=T_{\theta_{1}-}-K-k\left|A_{\theta_{1}-}-A_{1}\right|$, i.e. the starting point after the first intervention. Observe that from now on only $n-1$ interventions are possible, therefore $P_{T^{*}, A_{1}}\left(T_{t}^{\pi}>0\right.$ for all $\left.t \geq \theta_{1}\right) \leq v_{n-1}\left(T^{*}, A_{1}\right)$. In particular maximizing over $\theta_{1}$ and $A_{1}$ shows

$$
\varphi^{\pi}(x, A) \leq \mathcal{G} \varphi_{n-1}(x, A)=\varphi_{n}(x, A) .
$$

So we end up with $\varphi_{n}=\sup _{\pi \in \Pi_{n}} \varphi^{\pi}(x, A)$ and $\varphi_{n} \geq \varphi_{n-1}$ for all $n \in \mathbb{N}$.

Since we face an increasing sequence of functions which is bounded by the constant function equal to 1 we have that there exists a point-wise limit,

$$
\lim _{n \rightarrow \infty} \varphi_{n}(x, A)=v(x, A) \quad \text { for }(x, A) \in[0, \infty) \times[0, \infty) .
$$

From the definition of the sequence we have, together with bounded convergence, that

$$
v(x, A)=\lim _{n \rightarrow \infty} \mathcal{G} \varphi_{n-1}(x, A)=\mathcal{G} v(x, A) .
$$

It remains to show that $v=\varphi$. Because $\varphi_{n} \leq \varphi$ for all $n \in \mathbb{N}$ we have $v \leq \varphi$. For the opposite inequality we look at an $\varepsilon / 2$-optimal policy $\pi^{\varepsilon}=\left\{\left(\theta_{n}, A_{n}\right)\right\}_{n \in \mathbb{N}}$ such that $\varphi(x, A) \leq \varphi^{\pi^{\varepsilon}}(x, A)+\varepsilon / 2$. If $\pi^{\varepsilon}$ consists of finitely many interventions only, we are done. Therefore, assume it consists of infinitely many ones in the following. Define $\pi^{n} \in \Pi_{n}$ to be the policy using the first $n$ interventions of $\pi^{\varepsilon}$ only. Then we have

$$
T_{t}^{\pi^{\varepsilon}}=T_{t}^{\pi^{n}} \quad \text { for } 0 \leq t \leq \theta_{n},
$$

and from the Markov property of $\left(T_{t}^{\pi^{\varepsilon}}, A_{t}^{\pi^{\varepsilon}}\right)$ between interventions that

$$
\varphi^{\pi^{n}}(x, A)=P_{x, A}\left(\tau^{\pi^{\varepsilon}}>\theta_{n}\right) \mathbb{E}_{x, A}\left(\varphi^{0}\left(T_{\theta_{n}}^{\pi^{\varepsilon}}, A_{n}\right)\right) .
$$

Observe that on the set $\left\{\tau^{\varepsilon}=\infty\right\}$ there appear infinitely many jumps of size at least $K$ in the reserve process $T^{\pi^{\varepsilon}}$ defined in (1). From an argument part of the proof of Lemma 5 in Gaier et al. [13], we can deduce that $T_{t}^{\pi^{\varepsilon}} \rightarrow \infty$ as $t \rightarrow \infty$ on $\left\{\tau^{\pi^{\varepsilon}}=\infty\right\}$ and also that the sequence $T_{\theta_{n}}^{\pi^{\varepsilon}}$ can not be bounded as $\theta_{n} \rightarrow \tau^{\pi^{\varepsilon}}$. We get from (12)

$$
\lim _{n \rightarrow \infty} \varphi^{\pi^{n}}(x, A)=\varphi^{\pi^{\varepsilon}}(x, A) .
$$

Choosing $n$ large enough such that $\left|\varphi^{\pi^{n}}(x, A)-\varphi^{\pi^{\varepsilon}}(x, A)\right| \leq \varepsilon / 2$ we arrive at

$$
\varphi(x, A) \leq \varphi_{n}(x, A)+\varepsilon,
$$

by replacing $\varphi^{\pi^{n}}$ by $\varphi_{n}$. We obtain $v=\varphi$.

Remark 3.1 For the operator $\mathcal{G}$ we also have the property $\lim _{A \rightarrow \infty} \mathcal{G} f(x, A)=0$, which follows from the same arguments as for $M f$. When calculating $\varphi$, this asymptotic boundary condition is just of theoretical importance, since for large but fixed $A$ one would always try to get rid of some part of the investment position, as much as one can afford, through an immediate intervention. 
Remark 3.2 The characterization, stated in Theorem 3.3, gives also a guidance for calculating $\varphi$. Nevertheless this procedure would involve the solution of an optimal stopping problem at each step. In the next section we derive another characterization of $\varphi$ in terms of quasi-variational inequalities, which itself bears some difficulties since one would need to solve an associated free boundary value problem. In Sect. 4 we are going to illustrate that the combination of these two methods will lead to an efficient numerical procedure for approximating $\varphi$.

\subsection{Quasi-variational inequalities}

We start with deriving, for the moment heuristically, the so called quasi-variational inequalities (QVI) associated to our optimization problem given by (2). These inequalities can be seen as counterpart to the familiar Hamilton-Jacobi-Bellman equation in classical stochastic control theory.

Suppose that at $(x, A)$ with $\mathcal{D}(x, A) \neq \emptyset$ it is optimal to intervene, at such points we should have $M \varphi(x, A)=\varphi(x, A)$. Since immediate stopping may not be optimal in general, we have that $M \varphi \leq \varphi$ on $[0, \infty) \times[0, \infty)$.

Now suppose that it is not optimal to intervene in some neighborhood of a point $(x, A)$, then as for $\varphi^{0}$ assuming twice differentiability in $x$ and $A$ we shall have $\mathcal{L} \varphi(x, A)=0$. Combining the two observations we get

$$
\max \{\mathcal{L} \varphi(x, A), M \varphi(x, A)-\varphi(x, A)\}=0 .
$$

Formally, together with the boundary conditions, the QVI associated to $\varphi$ are given by

$$
\left\{\begin{array}{l}
\mathcal{L} w \leq 0 \\
w \geq M w \\
(w-M w) \mathcal{L} w=0 \\
\lim _{x \rightarrow \infty} w(x, A)=1 \\
\lim _{A \rightarrow \infty} w(x, A)=0 \\
w(0, A)=0
\end{array}\right.
$$

where $w$ denotes some suitable function and $\mathcal{L}$ is given by (5).

Since we can not expect that $\varphi \in \mathcal{C}^{2}([0, \infty) \times[0, \infty))$, we have to use a weaker concept of solution. The concept of viscosity solutions will be suited to characterize $\varphi$ in terms of the QVI (13). For an introduction to this concept see for example Fleming and Soner [10].

\section{Definition 3.4}

- A continuous function $v$ is a viscosity subsolution of (13) if for all $w \in \mathcal{C}^{2}$ such that $w-v$ attains a minimum equal to zero in $\left(x^{\prime}, A^{\prime}\right)$ we have

$$
\max \left\{\mathcal{L} w\left(x^{\prime}, A^{\prime}\right), \operatorname{Mv}\left(x^{\prime}, A^{\prime}\right)-v\left(x^{\prime}, A^{\prime}\right)\right\} \geq 0 .
$$

- A continuous function $u$ is a viscosity supersolution of (13) if for all $w \in \mathcal{C}^{2}$ such that $w-u$ attains a maximum equal to zero in $\left(x^{\prime}, A^{\prime}\right)$ we have 


$$
\max \left\{\mathcal{L} w\left(x^{\prime}, A^{\prime}\right), M u\left(x^{\prime}, A^{\prime}\right)-u\left(x^{\prime}, A^{\prime}\right)\right\} \leq 0 .
$$

- A function is a viscosity solution if it is a viscosity supersolution and a viscosity subsolution.

From [25, Lemma 7.3], or Krylov [21, p. 134], we have validity of the dynamicprogramming principle, which says that for a finite stopping time $\beta$ we have

$$
\varphi(x, A)=\sup _{\theta \leq \tau} \mathbb{E}_{x, A}\left(M \varphi\left(T_{\theta}^{A}, A_{\theta}\right) I_{\{\theta \leq \beta\}}+\varphi\left(T_{\beta}^{A}, A_{\beta}\right) I_{\{\beta<\theta\}}\right) .
$$

Theorem 3.5 The maximal survival probability $\varphi$ is a viscosity solution to (13).

Proof From Theorem 3.3 or (14) we have that $\varphi \geq M \varphi$ for all $(x, A)$.

Look at the subsolution property first and let $w$ be an appropriate test function. We need to prove the statement just on the set

$$
\mathcal{A}:=\{(x, A) \mid \varphi(x, A)>M \varphi(x, A)\} .
$$

Suppose $\varphi\left(x^{\prime}, A^{\prime}\right)-M \varphi\left(x^{\prime}, A^{\prime}\right)=\eta>0$ and define

$$
\mathcal{A}^{\eta}:=\left\{(x, A) \mid \varphi(x, A)-M \varphi(x, A)>\frac{\eta}{2}\right\}
$$

and set $\tau^{\eta}=\inf \left\{t \geq 0 \mid\left(T_{t}^{A^{\prime}}, A_{t}\right) \notin \mathcal{A}^{\eta}\right\}$, the first time leaving the set $\mathcal{A}^{\eta}$ when starting in $\left(x^{\prime}, A^{\prime}\right)$. We have that for $0 \leq t \leq \tau^{\eta}$ an intervention is not optimal and from (14) together with the assumptions on $w$ that

$$
w\left(x^{\prime}, A^{\prime}\right)=\varphi\left(x^{\prime}, A^{\prime}\right)=\mathbb{E}_{x^{\prime}, A^{\prime}}\left(\varphi\left(T_{\tau^{\eta}}^{A^{\prime}}, A_{\tau^{\eta}}\right)\right) \leq \mathbb{E}_{x^{\prime}, A^{\prime}}\left(w\left(T_{\tau^{\eta}}^{A^{\prime}}, A_{\tau^{\eta}}\right)\right) .
$$

Applying Itô's formula to $w$ gives

$$
0 \leq \mathbb{E}_{x^{\prime}, A^{\prime}}\left(\int_{0}^{\tau^{\eta}} \mathcal{L} w\left(T_{s}^{A^{\prime}}, A_{s}\right) d s\right)
$$

to see that the involved stochastic integrals are martingales and that ruin does not play a role here, one can cut off everything by stopping if the distance between $\left(x^{\prime}, A^{\prime}\right)$ and $\left(T_{t}^{A^{\prime}}, A_{t}\right)$ becomes too large such that the (then modified) function $w$ has compact support, cf. Theorem 2.1 of [25]. Now use $h \wedge \tau^{\eta}$ for $h>0$ and divide the above inequality by $0<\mathbb{E}_{x^{\prime}, A^{\prime}}\left(h \wedge \tau^{\eta}\right) \leq h$ and let $h \rightarrow 0$. By continuity of $\mathcal{L} w$ we get

$$
0 \leq \mathcal{L} w\left(x^{\prime}, A^{\prime}\right) .
$$

The supersolution property follows from the same ideas. From (14) we get (by taking $\theta=\tau$ )

$$
w=\left(x^{\prime}, A^{\prime}\right)=\varphi\left(x^{\prime}, A^{\prime}\right) \geq \mathbb{E}_{x^{\prime}, A^{\prime}}\left(\varphi\left(T_{\beta}^{A^{\prime}}, A_{\beta}\right)\right) \geq \mathbb{E}_{x^{\prime}, A^{\prime}}\left(w\left(T_{\beta}^{A^{\prime}}, A_{\beta}\right)\right),
$$

where $w$ is a suitable test function for the supersolution property. Now one can proceed as before using the stopping time 


$$
\beta_{n}=\inf \left\{t \geq 0 \mid d\left[\left(x^{\prime}, A^{\prime}\right),\left(T_{t}^{A^{\prime}}, A_{t}\right)\right] \geq \frac{1}{n}\right\} \wedge \frac{1}{n},
$$

instead of $\beta$ in the inequality above. Finally one gets

$$
\mathcal{L} w\left(x^{\prime}, A^{\prime}\right) \leq 0,
$$

which together with $M \varphi-\varphi \leq 0$ proofs the supersolution property.

Finally, we state a Verification Theorem linking a viscosity solution of (13) to $\varphi$.

Define $\phi(x, y)=\frac{1}{\pi} e^{-\left(x^{2}+y^{2}\right)}$ and let $u$ be a continuous, almost everywhere twice differentiable function, then for $n \in \mathbb{N}$

$$
v_{n}(x, y)=n^{2} \int_{-\infty}^{\infty} \int_{-\infty}^{\infty} u(x-s, y-t) \phi(n s, n t) d s d t
$$

we have that $v_{n}$ is twice differentiable, $\lim _{n \rightarrow \infty} v_{n}=u$ and also $\mathcal{L} v_{n}(x, y) \rightarrow \mathcal{L} u$ a.e., cf. [31].

Theorem 3.6 If $u:[0, \infty) \times[0, \infty) \rightarrow[0,1]$ is a continuous viscosity supersolution with $\lim _{x \rightarrow \infty} u(x, A)=1, \lim _{A \rightarrow \infty} u(x, A)=0, u(x, A)=0$ for $x \leq 0$ and is a.e. twice differentiable, then

$$
u \geq \varphi
$$

Proof Let $\pi=\left\{\theta_{i}, A_{i}\right\}_{i \in \mathbb{N}}$ be an arbitrary admissible policy. Since it can not be optimal to have two interventions at the same time we assume $\theta_{n}>\theta_{n-1}$ if $\tau^{\pi}>\theta_{n}$. We have for fixed $t>0$,

$$
\begin{aligned}
v_{n}\left(T_{t \wedge \tau^{\pi}}^{\pi}, A_{t \wedge \tau^{\pi}}^{\pi}\right)-v_{n}(x, A)= & \sum_{\theta_{n} \leq t \wedge \tau^{\pi}} v_{n}\left(T_{\theta_{n}-}^{\pi}, A_{\theta_{n}-}^{\pi}\right)-v_{n}\left(T_{\theta_{n-1}}^{\pi}, A_{\theta_{n-1}}^{\pi}\right) \\
& +\sum_{\theta_{n} \leq t \wedge \tau^{\pi}} v_{n}\left(T_{\theta_{n}}^{\pi}, A_{\theta_{n}}^{\pi}\right)-v_{n}\left(T_{\theta_{n}-}^{\pi}, A_{\theta_{n}-}^{\pi}\right) \\
\leq & \sum_{\theta_{n} \leq t \wedge \tau^{\pi}} v_{n}\left(T_{\theta_{n}-}^{\pi}, A_{\theta_{n}-}^{\pi}\right)-v_{n}\left(T_{\theta_{n-1}}^{\pi}, A_{\theta_{n-1}}^{\pi}\right) \\
& +\sum_{\theta_{n} \leq t \wedge \tau^{\pi}} M v_{n}\left(T_{\theta_{n}-}^{\pi}, A_{\theta_{n}-}^{\pi}\right)-v_{n}\left(T_{\theta_{n}-}^{\pi}, A_{\theta_{n}-}^{\pi}\right) .
\end{aligned}
$$

Taking expectations in the above inequality, using Itô's formula for the parts in the first sum and possibly a localization argument for the involved stochastic integrals and bounded convergence, we get

$$
\begin{aligned}
\mathbb{E}_{x, A}\left(v_{n}\left(T_{t \wedge \tau^{\pi}}^{\pi}, A_{t \wedge \tau^{\pi}}^{\pi}\right)\right) \leq & v_{n}(x, A)+\mathbb{E}_{x, A}\left(\sum_{\theta_{n} \leq t \wedge \tau^{\pi}} \int_{\theta_{n-1} \wedge t \wedge \tau^{\pi}}^{\theta_{n} \wedge t \wedge \tau^{\pi}} \mathcal{L} v_{n}\left(T_{s}^{\pi}, A_{s}^{\pi}\right) d s\right. \\
& \left.+\sum_{\theta_{n} \leq t \wedge \tau^{\pi}} M v_{n}\left(T_{\theta_{n}-}^{\pi}, A_{\theta_{n}-}^{\pi}\right)-v_{n}\left(T_{\theta_{n}-}^{\pi}, A_{\theta_{n}-}^{\pi}\right)\right) .
\end{aligned}
$$


Using bounded convergence and the properties of the sequence $\left\{v_{n}\right\}$ we arrive at

$$
\begin{aligned}
\mathbb{E}_{x, A}\left(u\left(T_{t \wedge \tau^{\pi}}^{\pi}, A_{t \wedge \tau^{\pi}}^{\pi}\right)\right) \leq & u(x, A)+\mathbb{E}_{x, A}\left(\sum_{\theta_{n} \leq t \wedge \tau^{\pi}} \int_{\theta_{n-1} \wedge t \wedge \tau^{\pi}}^{\theta_{n} \wedge t \wedge \tau^{\pi}} \mathcal{L} u\left(T_{s}^{\pi}, A_{s}^{\pi}\right) d s\right. \\
& \left.+\sum_{\theta_{n} \leq t \wedge \tau^{\pi}} M u\left(T_{\theta_{n}-}^{\pi}, A_{\theta_{n}-}^{\pi}\right)-u\left(T_{\theta_{n}-}^{\pi}, A_{\theta_{n}-}^{\pi}\right)\right) .
\end{aligned}
$$

Observe that in the present situation $v$ fulfills $\max \{\mathcal{L} u, M u-u\} \leq 0$ a.e.

Now we let $t \rightarrow \infty$ and use that $T^{\pi}$ either gets ruined or tends to infinity, which is based on the observations done in the proof of Theorem 3.3, to obtain

$$
P_{x, A}\left(\tau^{\pi}=\infty\right) \leq v(x, A) .
$$

Since the policy was arbitrary the result follows.

The next Corollary follows immediately.

Corollary 3.7 If an admissible policy $\pi^{*}$ generates an associated survival probability $\varphi^{*}$ which is an a.e. twice differentiable solution to (13), then $\varphi=\varphi^{*}$.

Remark 3.3 In Sect. 4 we are going to construct a sequence of admissible policies such that the associated survival probabilities form an increasing sequence. From step to step these survival probabilities are a.e. twice differentiable and approximate a solution to the QVI (13). Therefore using Corollary 3.7 we obtain an approximation of $\varphi$.

The asymptotic boundary condition $\lim _{A \rightarrow \infty} \varphi(x, A)=0$ for fixed $x$, which for the approximation is translated to $\bar{\varphi}(x, \bar{A})=0$ for $x \in[0, \bar{x})$ is just important for $0 \leq x \leq K$. For $x>K$ and $A$ close to $\bar{A}$, one always would do an immediate intervention to get away from the upper boundary for the investment position.

\section{Numerical approach}

We start with presenting the QVI linked to the sequence $\left\{\varphi_{n}\right\}_{n \in \mathbb{N}}$. Remember that the starting point is given by the pure survival probability $\varphi_{0}=\varphi^{0}$ and

$$
\varphi_{n}(x, A)=\mathcal{G} \varphi_{n-1}(x, A)=\max \left\{\varphi^{0}(x, A), \sup _{\theta<\tau} \mathbb{E}_{x, A}\left(M \varphi_{n-1}\left(T_{\theta}^{A}, A_{\theta}\right)\right)\right\} .
$$

Since one knows $\varphi_{n-1}$, we now face an optimal stopping problem with a given payoff function. By the same means as used in Sect. 3.2 one can show that $\varphi_{n}$ is (at least) a viscosity solution to

$$
\left\{\begin{array}{l}
\mathcal{L} w \leq 0 \\
w \geq M \varphi_{n-1} \\
\left(w-M \varphi_{n-1}\right) \mathcal{L} w=0 \\
\lim _{x \rightarrow \infty} w(x, A)=1 \\
\lim _{A \rightarrow \infty} w(x, A)=0 \\
w(0, A)=0
\end{array}\right.
$$


The exact calculation of $\varphi_{n}$ for several $n \in \mathbb{N}$ is an elaborate and time-consuming procedure. We therefore propose the usage of a so-called policy iteration procedure, see Chancellier et al. [7] or Bäuerle \& Rieder [3] for applications in finance. For discrete-time Markov models this method is also known as Howard's policy improvement algorithm, see Bäuerle and Rieder [4].

The idea of the method is explained in a few words: one proposes an admissible policy $\pi$ and calculates the corresponding survival probability $\varphi^{\pi}$. The second step generates an improved policy $\pi^{\prime}$ out of policy $\pi$. Using the new policy $\pi^{\prime}$, an immediate intervention in the point $(x, A)$ is carried out, if the difference $M \varphi^{\pi}(x, A)-\varphi^{\pi}(x, A)$ is positive. If the difference is negative, there will be no immediate intervention. In this way we generate two sets, an action (intervention) set $\mathcal{A}$ and a non-action (non-intervention) set $\mathcal{B}$.

The updated survival probability can now be calculated by solving numerically $\mathcal{L} \varphi^{\pi^{\prime}}$ on $\mathcal{B}$ and by setting $\varphi^{\pi^{\prime}}=M \varphi^{\pi}$ on $\mathcal{A}$.

We are going to implement this procedure on a bounded domain $[0, \bar{x}] \times[0, \bar{A}]$. The boundary points $\bar{x}$ and $\bar{A}$ are chosen sufficiently large, such that $\bar{\varphi}$, defined in Sect. 2.2, serves as a suitable approximation of $\varphi^{0}$.

The starting policy is given by choosing no intervention, the corresponding survival probability on $[0, \bar{x}] \times[0, \bar{A}]$ is given by $\bar{\varphi}$. From the proof of Lemma 2.2 , we can compute it numerically by using a discretization of the domain and by replacing the derivatives part of the operator $\mathcal{L}$ by appropriate finite differences.

Now we introduce the action set $\mathcal{A}_{1}$ and the non-action set $\mathcal{B}_{1}$ by

$$
\begin{aligned}
\mathcal{A}_{1} & :=\{(x, A) \in[0, \bar{x}] \times[0, \bar{A}] \mid M \bar{\varphi}(x, A)>\bar{\varphi}(x, A)\}, \\
\mathcal{B}_{1} & :=[0, \bar{x}] \times[0, \bar{A}] \backslash \mathcal{A}_{1} .
\end{aligned}
$$

The corresponding policy $\pi_{1}$ consists of at most one intervention which is given by

$$
\begin{aligned}
& \theta_{1}:=\inf \left\{t \geq 0 \mid\left(T_{t}^{A}, A_{t}\right) \notin \mathcal{B}_{1}\right\}, \\
& A_{1}:=\operatorname{argmax}\left\{M \bar{\varphi}\left(T_{\theta_{1}-}^{A}, A_{\theta_{1}-}\right)\right\},
\end{aligned}
$$

It turns out that the sets $\mathcal{A}_{1}$ and $\mathcal{B}_{1}$ can be stylized as given in Fig. 3. The red area corresponds to the non-action set and the dashed line corresponds to $A^{*}$, the optimal constant amount to hold in the risky asset for the problem without transaction costs.

Therefore the associated survival probability $\varphi^{\pi_{1}}$ can be computed by

$$
\varphi^{\pi_{1}}(x, A)=M \bar{\varphi}(x, A) \quad \text { for }(x, A) \in \mathcal{A}_{1},
$$

and by determining a solution to $\mathcal{L} \varphi^{\pi_{1}}(x, A)=0$ for $(x, A) \in \mathcal{B}_{1}$ with the boundary conditions given by

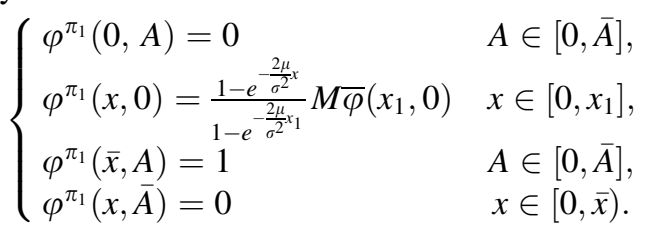

The point $x_{1}=\inf \left\{x \geq 0 \mid(x, 0) \in \mathcal{A}_{1}\right\} \geq K$ is the smallest point on the $x$-axis which lies in the action set. 
Fig. 3 Stylized action and nonaction sets

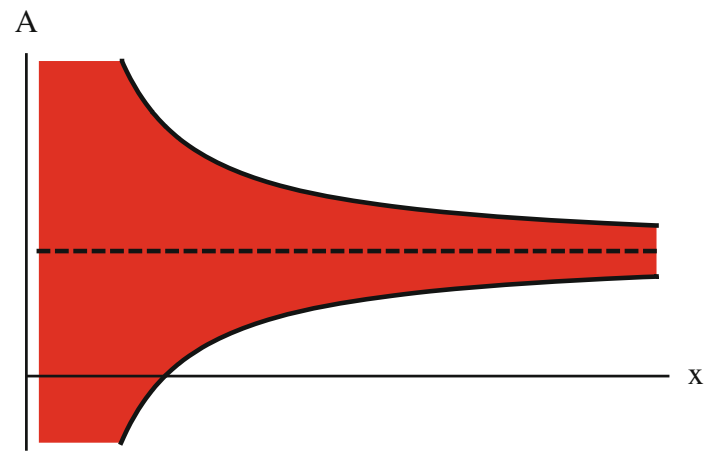

Now one iterates this procedure by replacing $\bar{\varphi}$ by $\varphi^{\pi_{n-1}}$ in the steps described above, for generating $\varphi^{\pi_{n}}$ out of $\varphi^{\pi_{n-1}}$. The application of the proposed algorithm generates a sequence of survival probabilities and admissible investment policies. It is stopped if the improvement of $\varphi^{\pi_{n}}$ over $\varphi^{\pi_{n-1}}$ becomes marginal.

Remark 4.1 For showing that the sequence $\left\{\varphi^{\pi_{n}}\right\}_{n \in \mathbb{N}}$ is increasing and really constitutes an improvement, we can follow the arguments given by Bäuerle and Rieder [3]. We observe, when using a maximum principle [9, p. 332] for $\varphi^{\pi_{1}}$ on $\mathcal{B}_{1}$, that $\varphi^{\pi_{1}}$ attains its maximum and minimum at the boundaries of the non-action set, $\delta \mathcal{B}_{1}$. Note, because on $\{(x, \bar{A}), \mid 0 \leq x \leq \bar{x}\} \cap \delta \mathcal{B}_{1}$ there may still be a discontinuity in the boundary condition, we need at first to approximate the boundary condition by a continuous function to guarantee continuity of $\varphi^{\pi_{1}}$ at the boundary. But this changes nothing in the line of argumentation, since one could replace a classical maximum principle by one used for viscosity solutions.

Since $\varphi^{\pi_{1}}(x, A)=M \bar{\varphi}(x, A)$ on $\delta \mathcal{B}_{1}$ we achieve a higher boundary value for $\varphi^{\pi_{1}}$ than for $\bar{\varphi}$, by construction of the action set $\mathcal{A}_{1}$. Therefore $\varphi_{1} \geq \bar{\varphi}$ and an induction argument shows that $\varphi^{\pi_{n}} \geq \varphi^{\pi_{n-1}}$.

Remark 4.2 At step $n$ of the iteration at most $n$ interventions are done, the policy $\pi_{n}=\left\{\left(\theta_{k}, A_{k}\right)\right\}_{k=1}^{n}$ is given by

$$
\begin{aligned}
\theta_{k} & :=\inf \left\{t \geq \theta_{k-1} \mid\left(T_{t}^{\pi_{n}}, A_{t}^{\pi_{n}}\right) \notin \mathcal{B}_{n+1-k}\right\}, \\
A_{k} & :=\operatorname{argmax}\left\{M \varphi^{\pi_{n-k}}\left(T_{\theta_{k}-}^{\pi_{n}}, A_{\theta_{k}-}^{\pi_{n}}\right)\right\} .
\end{aligned}
$$

It says that the first intervention, which is going to be applied, is the one determined in the last step of the iteration.

If $\varphi^{\pi_{n}} \sim \varphi^{\pi_{n-1}}$, one has approximately generated a fixed point of the operator $\mathcal{G}$. Therefore the policy $\pi^{*}$ corresponding to

$$
\begin{aligned}
& \theta_{k}:=\inf \left\{t \geq \theta_{k-1} \mid\left(T_{t}^{\pi^{*}}, A_{t}^{\pi^{*}}\right) \notin \mathcal{B}_{n}\right\}, \\
& A_{k}:=\operatorname{argmax}\left\{M \varphi^{\pi_{n}}\left(T_{\theta_{k}-}^{\pi^{*}}, A_{\theta_{k}-}^{\pi^{*}}\right)\right\},
\end{aligned}
$$

suggests a reasonable approximation of the optimal policy. 
As an illustration of the method we perform some iterations for the set of parameters given in Table 1. The corresponding plots of step-wise improved survival probabilities are given in Figs. 4 and 5. The associated investment policy, expressed through their action (indicated as positive values equal to the optimal intervention) and non-action (indicated as zero values) sets is displayed in the right graphic of 5. A detailed interpretation of the numerical results will be given in the following section.

\section{Interpretation of results: conclusion}

In the present manuscript we incorporated transaction costs into the problem of maximizing an insurer's survival probability by controlling the amount of money invested in a risky asset. This is an additional step towards a practical application of results on optimal investment for an insurer achieved from research in the context of risk theory. The used diffusion model, irrespective of its simplicity, has the big advantage that all involved parameters have a clear interpretation. In this way it is possible to separate effects on the optimal investment policy and the associated survival probability by cause, i.e. one can observe exactly which changes in the behaviour of the risky asset generate changes in the survival probability and investment policy. Modelling the survival probability as a bivariate function of the total surplus and the amount of money invested allows to interpret the initial investment position as the outcome of a previously used investment policy. Since this old policy may not have been optimal, the first intervention can be used to correct this misleading initial position. From the numerical results it is obvious that this intervention, as calculated by the first intervention, has the biggest effect on improving $\varphi^{0}$.

The complete theoretical characterization allows to describe a method to compute the maximal survival probability quite easily by iteration. The used extended solution concept in Sect. 3.2 for characterizing the survival probability as a solution to the quasi-variational inequalities is just a technical issue, since a priori one can hardly make any statement about the regularity of a solution to these inequalities. When computing the maximal survival probability in Sect. 4, this technical difficulty does not play a big role anymore; one can build up the solution by iteratively calculating solutions to partial differential equations. However the theoretical results are important to back up the numerical results.

Although the obtained results have some theoretical flavour, they nevertheless point out some guidelines on how to behave in different situations faced in practice. In the following we are going to summarize the results from qualitative points of view.

Table 1 Parameters for iteration procedure

\begin{tabular}{lllllll}
\hline$\mu$ & $\sigma$ & $a$ & $b$ & $K$ & $k$ & $A^{*}$ \\
\hline 0.1 & 0.4 & 0.15 & 0.25 & 0.1 & 0.02 & 1.067 \\
\hline
\end{tabular}



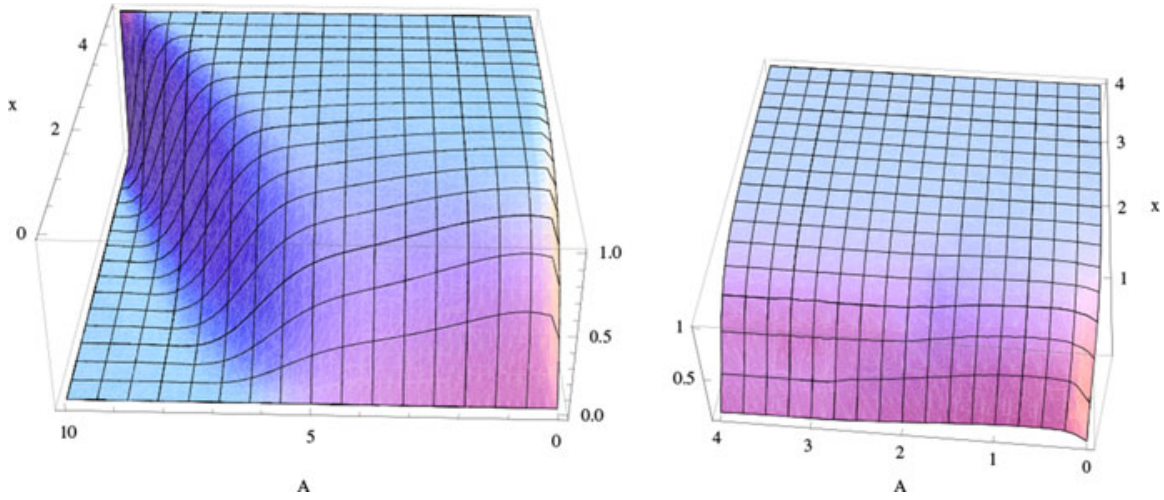

Fig. $4 \varphi(x, A)$ without (left) and with one intervention (right) for parameters from Table 1
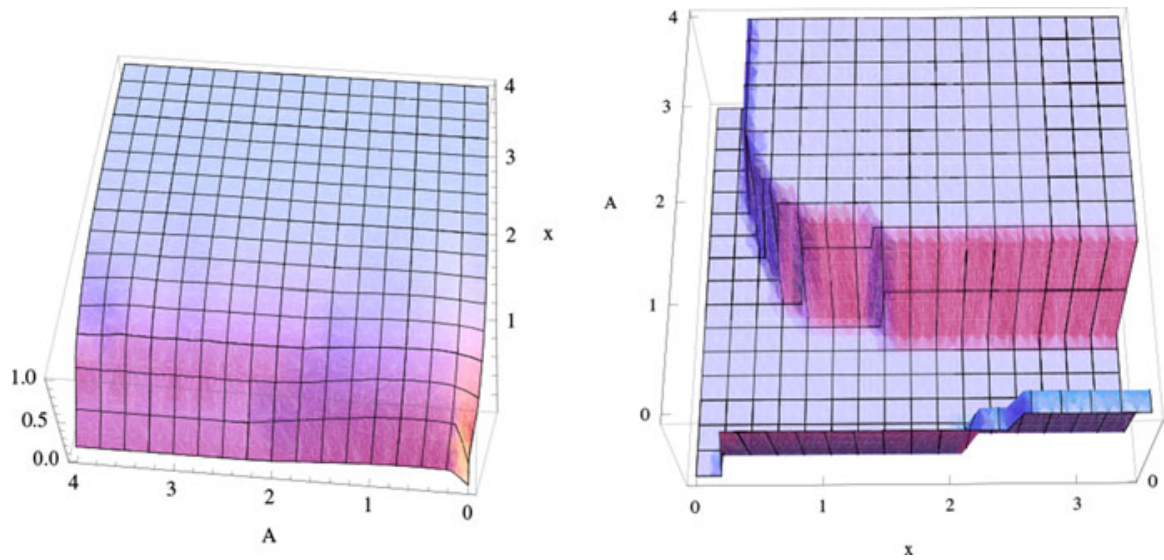

Fig. $5 \varphi(x, A)$ with 3 (left) interventions and policy (right) for parameters from Table 1

\subsection{Optimal policy}

When comparing the outcome of the numerical method for several parameter sets, we obtained that the calculated results concerning the behaviour of the optimal investment policy are very stable. This allows us to draw some quite general conclusions on the form of the optimal investment policy.

The crucial quantity is still given by the optimal amount $A^{*}$ to be invested for the problem without transaction costs. The optimal policy for the problem including transaction costs is given by a certain action set $\mathcal{A}^{*}$ and a certain non-action set $\mathcal{B}^{*}$. Recall that, as long as the bivariate process $\left(T_{t}^{\pi^{*}}, A_{t}^{\pi^{*}}\right)$ is in the set $\mathcal{B}^{*}$ there is no intervention, if it enters $\mathcal{A}^{*}$ one immediately adapts the investment position. The action set can be interpreted as the set of non-acceptable investment positions, whereas the non-action set consists of acceptable positions. 
Fig. 6 Stylized optimal interventions for some combinations of $(x, A)$

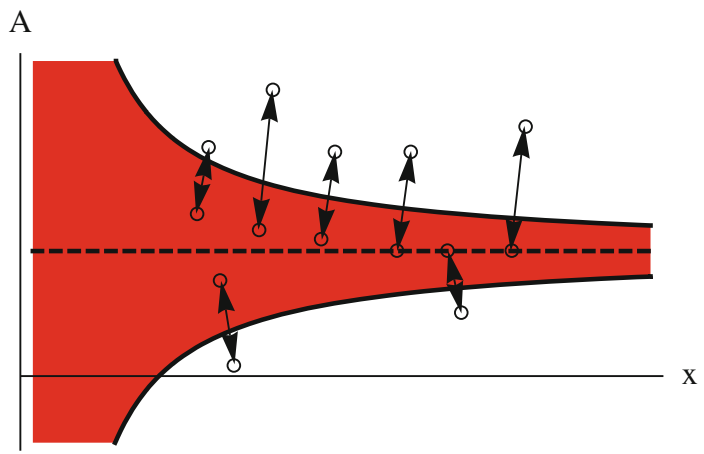

A striking observation is the structure of the non-action set $\mathcal{B}^{*}$, as it is a connected area, which is roughly symmetric around the constant $A^{*}$ and for large $x$ the lower and upper boundaries of $\mathcal{B}^{*}$ converge to $A^{*}$. The optimal new investment positions are - as soon as on can afford this type of restructuring very close to the value $A^{*}$, cf. Fig. 6 , where arrows connect the pre- and postintervention positions for some points $(x, A)$. For initial positions $(x, A)$ with small $x$, it is possible that the investment position can not be changed to $A^{*}$ because of a too high value of transaction costs which would be equal to $K+$ $k\left|A-A^{*}\right|$. By the structure of $\mathcal{B}^{*}$, we get that for large $x$ the maximal survival probability with transaction costs $\varphi$ is quite close to $\varphi^{B}$, the classical survival probability using optimal investment. This observation tells us that, instead of continuously rebalancing the investment position, in the presence of transaction costs one just intervenes if the investment position grows too far away from its optimal position. Since for large initial capital the non-action set becomes very narrow, there will be more interventions, in short time intervals than for small initial capital, which keep the investment component close to $A^{*}$. For small initial capitals the positioning of the first intervention decides if investment is advantageous or not.

Another observation is the stability of the optimal action and non-action sets across the iterations. Actually it turns out that the optimal intervention set computed in the first iteration almost matches the one from the second iteration and also performing several steps of the iteration does not show any substantial changes, cf. Fig. 6. Therefore, already the first determined action and non-action sets yield an approximation of a reasonable investment policy at later iterations.

\subsection{Optimal solution $\varphi$}

The results of Sect. 4 show that in principle just a few adaptations are enough to considerably improve the resulting survival probability. A convenient consequence is that the execution of the investment policy can be done in a relaxed manner. Just for small initial capital an accurate behaviour is really important, since wrong investment positions have severe consequences. 


\subsection{Still need for investment}

Actually, the results of the present paper also reflect an important aspect in the present economic environment, that is, namely, the need of reduction (or even liquidation) of the investment position. Nevertheless, optimal investment (possibly on a smaller scale) still has a positive effect on an insurance portfolio's safety. In the presented model this fact is reflected in the behaviour of $A^{*}$, which is always positive but decreases with respect to the volatility of the asset. Since the non-action set is centered by this constant, the optimal policy with transaction costs also takes smaller investment positions in the presence of high risk in the financial market. The importance of changing the investment position can be seen quite clearly by comparing the plots in Fig. 4 and the plots in Fig. 5, even only one optimal intervention shifts the survival probability to a much higher level.

Acknowledgments The author would like to thank Valentina Troncale for support with the implementation of the numerical procedure which is used for the generation of the depicted plots and Hansjörg Albrecher for several discussions on the topic.

\section{References}

1. Asmussen S, Albrecher H (2010) Ruin probabilities, 2nd edn. World Scientific, River Edge

2. Azcue P, Muler N (2009) Optimal investment strategy to minimize the ruin probability of an insurance company under borrowing constraints. Insur Math Econ 44(1):26-34

3. Bäuerle N, Rieder U (2012) Control improvement for jump-diffusion processes with applications to finance. Appl Math Optim 65(1):1-14

4. Bäuerle N, Rieder U (2011) Markov decision processes with applications to finance. Springer, Heidelberg

5. Belkina T, Hipp C, Luo S, Taksar M (2013) Optimal constrained investment in the Cramér-Lundberg model. Scand Act J. doi:10.1080/03461238.2012.699001

6. Browne S (1995) Optimal investment policies for a firm with a random risk process: exponential utility and minimizing the probability of ruin. Math Oper Res 20(4):937-958

7. Chancelier J-P, Messaoud M, Sulem A (2007) A policy iteration algorithm for fixed point problems with nonexpansive operators. Math Methods Oper Res 65(2):239-259

8. Eisenberg J (2010) On optimal control of capital injections by reinsurance and investments. B1 DGVFM, 31(2):329-345

9. Evans LC (1998) Partial differential equations. American Mathematical Society, Providence

10. Fleming WH, Soner HM (1993) Controlled Markov processes and viscosity solutions. Springer, New York

11. Frolova A, Kabanov Y, Pergamenshchikov S (2002) In the insurance business risky investments are dangerous. Finance Stoch 6(2):227-235

12. Gaier J, Grandits P (2002) Ruin probabilities in the presence of regularly varying tails and optimal investment. Insur Math Econ 30(2):211-217

13. Gaier J, Grandits P, Schachermayer W (2003) Asymptotic ruin probabilities and optimal investment. Ann Appl Probab 13(3):1054-1076

14. Hipp C, Plum M (2000) Optimal investment for insurers. Insur Math Econ 27(2):215-228

15. Hipp C, Plum M (2003) Optimal investment for investors with state dependent income, and for insurers. Finance Stoch 7(3):299-321

16. Hipp C, Schmidli H (2004) Asymptotics of ruin probabilities for controlled risk processes in the small claims case. Scand Actuar J 5:321-335

17. Iglehart DL (1969) Diffusion approximations in collective risk theory. J Appl Probab 6:285-292

18. Jost J (2002) Partial differential equations. Springer, New York

19. Kalashnikov V, Norberg R (2002) Power tailed ruin probabilities in the presence of risky investments. Stoch Process Appl 98(2):211-228 
20. Korn R (1998) Portfolio optimization with strictly positive transaction costs and impulse control. Finance Stoch 2(2):85-114

21. Krylov NV (1980) Controlled diffusion processes. Springer, New York

22. Merton RC (1969) Lifetime portfolio selection under uncertainty: the continuous time case. Rev Econ Stat 51:247-257

23. Merton RC (1971) Optimum consumption and portfolio rules in a continuous-time model. J Econ Theory 3(4):373-413

24. Øksendal B (1998) Stochastic differential equations, 5th edn. Springer, Berlin

25. Øksendal B, Sulem A (2005) Applied stochastic control of jump diffusions. Springer, Berlin

26. Paulsen J (1993) Risk theory in a stochastic economic environment. Stoch Process Appl 46(2):327-361

27. Paulsen J, Gjessing HK (1997) Ruin theory with stochastic return on investments. Adv Appl Probab 29(4):965-985

28. Schmidli H (2002) On minimizing the ruin probability by investment and reinsurance. Ann Appl Probab 12(3):890-907

29. Schmidli H (2004) Diffusion approximations. In: Teugels JL, Sundt B (ed) Encyclopedia of actuarial sciences, vol 1. Wiley, Chichester, pp 519-522

30. Schmidli H (2008) Stochastic control in insurance. Springer, New York

31. Wheeden RL, Zygmund A (1977) Measure and integral. Marcel Dekker Inc., New York 